\title{
Potential Problems and Uses for Artificial Intelligence in Clinical Medicine Eisuke Hanada
}

Department of Information Science, Faculty of Science and Engineering, Saga University, Saga, Japan

\section{Abstract}

Publication History:

Artificial intelligence (AI) is being widely used, with trial systems already in use in the medical field, where there are high expectations for its potential for use in the future. Studies have shown that AI diagnosis is almost as accurate as that of human experts. However, problems with the use of AI in clinical medicine must be identified and carefully examined to insure that it can be used safely. Herein, I illustrate various concerns related to the use of $\mathrm{AI}$ and show how it can be put to practical use in clinical medicine. The concerns about the use of $\mathrm{AI}$ include the following: differences in expectations for AI, failure to correctly diagnose disease because of a lack of data available for learning, and over dependence by physicians using it to make a diagnosis. AI can also be useful to hospital staff other than physicians. We should not leave the business of medicine and healthcare to AI systems, but use it to assist with decision-making and to raise labor efficiency and effectiveness.
Received: February 03, 2020

Accepted: March 02, 2020

Published: March 04, 2020

\section{Keywords:}

Artificial intelligence, Clinical medicine, Specialty, Q\&A system

\section{Introduction}

Systems using Artificial Intelligence (AI system) are being widely used with various degrees of success. Some trial systems for clinical use are already in place [1-4], and studies have shown that AI diagnosis is almost as accurate as that of human experts [5]. The practical use of AI systems in the medical field is growing, and can be expected to grow even more rapidly in the future. However, certain problems have been identified in the use of AI for clinical medicine, and others will surely be found in the future. Here, I illustrate some of the problems and how AI systems can be put to practical use in clinical medicine.

\section{Expectations for AI from Various Perspectives}

One of the main problems about the use of AI in clinical medicine is a difference of expectations, with developers, physicians, and patients addressing the issue from different viewpoints. The following are often stated by people with a layman's perspective, and seeing AI in this simplistic manner can be problematic in itself.

1. AI may be superior to humans.

2. The system always outputs the same result when the same data is input

3. AI never makes mistakes.

A human physician makes a diagnosis using his/her range of knowledge at any given time. Compared with AI, which has access to a wider range of information, doctors making a diagnosis can only do and analyze the results of examinations that follow their training in their often quite narrow specialty. This can result in a mistaken diagnosis that could have been correctly made with a broader range of information. It is human get tired, become absent-minded, and to sometimes make mistakes and it is difficult for humans to use the full range of their knowledge at all times.

In contrast, AI can quickly and accurately judge all the possibilities at all times, using all of their learned knowledge, without getting tired. Because disease classifications and guidelines have multiplied in recent years, accurate diagnosis has become increasingly difficult. After a diagnosis is made, a wide range of treatment options are available, $\mathrm{AI}$ has the advantage of having access to the latest information on the full range of possible diagnoses and treatments, which makes it an important, if not indispensible, tool for assisting doctors with their diagnosis and choice of treatment regimen. However, the rapid, complex changes mean that the AI system must be constantly updated, which if not done in a timely and careful manner can be problematic.
From a developers' viewpoint, AI is limited because it can search only in its learned data. This means that AI cannot associate knowledge from domains that it has not learned or does not have access to.

\section{Performance of AI in Clinical Diagnosis}

Although there are differences by country, a physician's license is granted on the premise of having studied the fundamentals of medicine across all domains. However, almost all physicians have a specialty as their main focus, and physicians who work in large hospitals tend to be highly (and narrowly) specialized. Often when building an AI system for use in clinical medicine, one or several of these highly specialized physicians take the initiative. In such cases, AI will tend to learn only knowledge based on the specialty of the physician(s) involved. This means that such specialized AI will overlook the existence of illnesses that have not had the chance to learn or that are out of the scope of the AI system. AI on which two or more clinical departments have co-operated is seldom seen.

Some symptoms are associated with a broad range of diseases and may differ by patient, even patients with the same disease. An example of a problematic case is a patient who has had their primary disease diagnosed but who newly contracts another disease, the symptoms of which may differ from those of other patients with the same primary disease. In such cases, AI would probably not be useful for assisting with diagnosis or treatment. In contrast, for some diseases the mechanism is clear and differentiation using symptoms is easy. The use of AI could be problematic in cases where the correct disease is not output because there were too few candidate diseases input. Such cases show the potential weakness of using AI for the screening of patients and making the final determination of a patient's disease. As I stated previously, I think that highly specialized AI can be used for assisting with detailed diagnosis after screening, but the disease candidates it has available to choose from must be wide ranging and the amount of data limited to that which is necessary. Providing AI with the data necessary to achieve this high level of performance is

"Corresponding Author: Prof. Eisuke Hanada, Department of Information Science, Faculty of Science and Engineering, Saga University, Saga, Japan; E-mail: hanada@cc.saga-u.ac.jp

Citation: Hanada E (2020) Potential Problems and Uses for Artificial Intelligence in Clinical Medicine. Int J Comput Softw Eng 5: 154. doi: https://doi. org/10.15344/2456-4451/2020/154

Copyright: (C) 2020 Hanada. This is an open-access article distributed under the terms of the Creative Commons Attribution License, which permits unrestricted use, distribution, and reproduction in any medium, provided the original author and source are credited. 
possible, but realizing such a system will involve a number of very difficult problems, especially concerning data acquisition.

The field of medicine is evolving at an incredible pace, accompanied by rapidly changing diagnostic and treatment techniques. New treatment approaches can be seen being used alongside older treatment modalities for the same disease. Even with a new AI system, it is important that it be flexible and continuously updated to meet these evolving needs

\section{Dependency on AI}

There is widespread thinking that "AI makes a physician's burden lighter", which is essentially correct; however, too much dependency on AI raises the potential for conflict with the concept of physician autonomy. Over dependency could negatively impact the ability of physicians to effectively care for their patients. It is the physician who must take the responsibility for a patient's treatment, and often life and death decisions must be made. The Ministry of Health, Labour and Welfare of Japan has stated clearly "AI can only be used to support a physician. It does not have the right to make decisions" [6]. A relationship between a physician and AI must be maintained in which the physician makes the final decision, without total dependence on the AI output, but using it only for support.

\section{By whom can AI be Effectively Used?}

\section{AI use by staff other than physicians}

I have discussed the problems physicians confront with AI. Except for small clinics, co-medical and other staff are also important to the functioning of hospitals. In Japan, there are over 20 kinds of state qualification for people working in the medical profession. Sections that are not strictly medical manage the hospital building, facilities, equipment, medical devices, computer and network systems, and do office work.

For staff such as nurses and pharmacists, it is easy to see how AI could be used to support them. When outputs and inputs are integrated in a system, AI is easy to use. Q\&A systems are good examples of a suitable use for AI. In addition, judgments based on work that has clear operating procedures are also easily realizable by AI. However, when an AI system is built to include the work of two or more kinds of staff, the system will become so large that it increases the difficulty of maintenance and can slow the system to such a degree that it is not effective. Because of the wide differences in the work of the various staff sections, I think that the content a single AI should be expected to learn needs to be limited to a single category or operation for each kind of staff.

Reducing the load of the staff leads to a reduction of the physicians' workload. This load reduction should not be used only to reduce working hours, but should be used to increase face time with patients.

\section{Possible AI uses for patients}

What are the possible uses, other than Q\&A systems, of AI for patients? An example is one that can predict a patient's disease course using AI with deep learning. The duration of hospitalization has been getting shorter in large Japanese hospitals and patients may access multiple hospitals and clinics. This makes it difficult for any one hospital to follow patients and their needs in terms of the course of treatment or recovery. For long-term hospitalized patients who are discharged to their homes or to health care facilities, such as for the elderly, the long-term course of the patient may also be difficult to follow. By integrating hospital and clinic systems, patient data from multiple sources can be used as learning data for AI. The planning of this type of AI system requires extensive collaboration to insure that all relevant data is acquired. However, an integrated system would be of great benefit by making it possible to better follow and predict changes in the long-term course of treatment and follow-up and to reduce and prevent personal and medical accidents. The aging of the population has progressed rapidly in Japan, and most countries are following a similar course. Realizing AI technology that can be used to ameliorate the medical problems that face an aging population will be of great benefit.

\section{Conclusion}

In this paper, while illustrating problems related to AI use in clinical medicine, I have shown practical ways for its use from various perspectives. We should not leave the decisions of medicine and healthcare solely to AI systems, but should use them to raise medical and labor efficiency and effectiveness. To best utilize the potential of AI for medical practice, it will be important to be selective about how it is used and to be careful about the selection of data for AI learning.

\section{Competing Interests}

The author declare that there is no competing interests regarding the publication of this article.

\section{References}

1. Kapoor R, Walters SP, Al-Aswad LA (2019) The current state of artificial intelligence in ophthalmology. Surv Ophthalmol 64: 233-240.

2. Kikuchi A, Kawakami T (2018) Future of Artificial Intelligence and Nuclear Cardiology. Annals of Nuclear Cardiology 4: 79-82.

3. Ting DSW, Pasquale LR, Peng L, Campbell JP, Lee AY, et al. (2019) Artificial intelligence and deep learning in ophthalmology. Br J Ophthalmol 103: 167-175.

4. Nakajima K, Matsuo S, Wakabayashi H, Yokoyama K, Bunko H, et al. (2015) Diagnostic Performance of Artificial Neural Network for Detecting Ischemia in Myocardial Perfusion Imaging. Circ J 79: 1549-1556.

5. Thorey V, Hernandez AB, Arnal PJ, During EH (2019) AI vs Humans for the diagnosis of sleep apnea. Annual International Conference of the IEEE Engineering in Medicine and Biology Society (EMBC).

6. The Health Policy Bureau, Medical Professions Division, the Ministry of Health, Labour and Welfare (Japan) (2018) The relationship between the use of programs which support diagnosis, treatment, etc. using artificial intelligence (AI), and the regulation of Article 17 of the Medical Practitioners' Act. 